\title{
Reframing online shopping through innovative and organic user-oriented design
}

\author{
Anita Greenhill
}

Manchester Business School, University of Manchester

\section{Gordon Fletcher}

Salford Business School, University of Salford

\section{x.1 Exploring Blogshops}

The structure and form of the Web is defined by specific design elements; its protocols, the scope of acceptable file formats and the capability of clients. These elements are intentionally minimal constraints but nonetheless structure what can be achieved "through" the Web. With the increasingly standardised abilities of Web clients and wider application of accessible design principles increasingly emphasis shifts from the problem of creating and developing monolithic software systems towards concern for the integration and configuration of existing systems to meet specific social and cultural needs. The free availability of, for example, open source content management systems and blogging applications has increased the ability for individuals and closely geographically constrained highly specialised interest groups to more rapidly represent and express themselves through the Web. Importantly, the abilities provided by prebuilt existing systems enables greater focus on reflecting and capturing the peculiar cultural sentiments of special interests. 
This chapter explores innovative and organic user-oriented design found in the grassroots ecosystem management (GREM) of Singaporean Blogshops. The intersection of Web2.0 integration tools and freely available open source systems coupled with self-motivated shopping practices introduces the capacity for direct conversation and immediate expression in social media that, in turn, produces unexpected forms of empowerment (Beer and Burrows 2007). The research presented here reveals how young Singaporean women are utilising blogging systems to fulfil their desires to possess identifiable "brand name" objects of fashion. Satisfying these desires requires exchange practices and communication that extend and increases their personal and financial capability to obtain goods. These practices are conducted in parallel with conventional commercial activities in the sense that these women maintain everyday desires for recognisable brand names and employ the rhetoric and imagery familiar to a commercially oriented style sentiment. However, the mechanism by which they seek to obtain these goods is undertaken outside what is seen as conventional shopping practice. The bottom-up motivations of Blogshops illustrates how user-oriented design does not necessitate originality and the personal desire to accumulate currently fashionable items mitigates towards reuse and repurposing of conventional mainstream imagery and rhetoric in conjunction with localised vocabulary and practices.

By utilising empirical data gathered from a selection of Blogshops we present contemporary examples of polymorphic and interactive design practices (Cooley 1987). Our research asks whether online design practices necessarily adopts a separation of designer and developer from use and users and whether online design practice is always, even necessarily, audience-focused. We argue that Blogshops consciously represent the interests of the individual Blogshop hosts designed by them on their terms and in a style that appeals primarily to them personally. This perspectives enables the hosts to be the designers for a primary audience of one; themselves. This observation is albeit unsurprising with the context of blog authorship but counter-intuitive to the commercial 
need for a buying audience. The Blogshops, however, remain relevant and even enticing for a secondary audience, of other teenage Singaporean girls, because of shared (micro) cultural understandings and worldviews. Blogshops cannot be seen as directly attentive to the requirements of a clearly articulated mass audience or alternatively 'simply' be seen as examples of unconsciously ironic anti-design (Whiteley 1993).

The research presented in this chapter problematises the classification of user in distinction from that of designer and the implication that these categories could be mutually exclusive or readily differentiated aspects of an individual experience, cultural experience or capacity. The term "bottom up" participation has previously been utilised primarily in relation to governance, democracy and community oriented organisational practices. Weber (2003) in referring to grassroots ecosystem management (GREM) states, "instead of a system premised on hierarchy and commands from above, GREM emphasizes nonhierarchical, place-based networks...expertise is substantially redefined to include local expertise and community-based folk knowledge. Canas (2009) reminds us that the human and the technology depend on each other and cannot be studied separately. He states that 'the term humanmachine interaction refers to the design of the interface where a person and a machine interact during the execution of a task'" $(2009,55)$. Weber contends that designers work in either academic or industrial environments, where an academic's primary role is to explain interactive behaviour and industry-based designers are commissioned to design for a need-specific context by providing solutions, for example, to reduce costs or obtain benefits within short-term projects. The hosts as the designers of Blogshops are neither academics nor situated in a conventional industrial environment but instead exist between a range of roles and understanding as the creators of home-grown eCommerce ventures. The examples drawn from this study present everyday adaptation of Web technologies, where conventional ideas of human-computer design are reframed and reduced to a scale that benefits individual purposes and motivations. 
Blogshops are an online shopping phenomenon that can currently be most clearly observed in Singapore. We have previously suggested that the Blogshop phenomenon emerged through the symbiotic relationship of technology adaptation and design with specific geographic conditions including public transport infrastructure (Greenhill and Fletcher 2009). The public visibility and clarity of Blogshop exchange events constitutes Singapore as an experimental viewport for understanding a partially Webbased practice in situ. Similar online shopping sites that deviate from conventional perspectives of eCommerce design and implementation can already be identified within activities based in the United Kingdom (UK) and United States (USA). However, Singaporean Blogshops, as the primary location of the most completely articulated concept expressed through well-established sites presenting the widest variety of goods, frames contemporary technology-mediated experiences within a specific and identifiable social and cultural group. Unlike many discussions concerning Web technologies and the general level of uncertainty surrounding the identity of its users (Beer 2008), Blogshops can be precisely identified within the domain of female teenagers (usually around the age of sixteen) who are 'middle class' college students living primarily in the outlying suburbs of Singapore. This group are thoroughly immersed within a particular technoscape (Crang et al. 2007) that incorporates daily engagement with innovative and organic design practices, social networking and eCommerce as well as the more mundane day-to-day practicalities of attending a college and constant exposure to a globalised mainstream fame-obsessed culture. The influences of this environment coalesce within the form of Blogshops. While a Blogshop ostensibly uses the blog to sell fashion objects for personal financial gain such a primarily economic observation obscures more subtle design and cultural issues. Blogshops adopt a unique iterative and polymorphic design sentiment by drawing upon a rich assemblage including Web and mobile technologies, innovative and creative design skills, easy access to major international export centres of consumable goods, access to 'middle class' levels of disposable income, Singaporean-specific cultural knowledge and an efficient urban public transport system. The combination of available 
open source technology with a desire to sell for specific personal and cultural motivations presents a critique of prescriptive design practices (He and King 2008) including, for example, received understanding of what is necessary to enable an eCommerce payment system or the stated requirement for Web sites to have mechanisms 'designed in' that cultivates a purchaser's trust (Jarvenpaa et al. 1998). By largely ignoring the presence of an external audience hosts overcome these apparently essential design concerns by effacing the distinction between the role of user from that of developer (Greenbaum and Kyng 1991).

\section{x.2 Designing Blogshops}

Blogshops are readily defined from a technology point-of-view as virtual shop fronts that utilise third party hosted blogging systems. But this neat statement belies the complexities of Blogshop practice. Blogshops use multiple blogs to construct different "departments" for separate classes of items as well as specific events including sales and sprees. A hallmark of the most established Blogshops are the number of individual blogs being maintained and are part of their evolving design from a conventionally purposed weblog. However, Blogshop departments are themselves critiques of conventional shopping and its rarely questioned classifications. For example babys-candy-new.blogspot.com offers departments such as 'pretty', 'instock' and 'itemssold'. Utilising multiple blogs for what is ostensibly a single purpose could be dismissed as naivety on the part of the hosts and the apparent naivety of the hosts is itself a hallmark of Blogshops. However, the subtlety and regularity of the multiple blog form, as well as other aspects of hosts' activities, reveals a collective deeper understanding of the workings of blogging systems and the application of effective methods for obtaining maximum personal benefit from their exploitation. As a single web page a single blog can only accommodate a single thread of discussion whereas the better established Blogshops are constantly engaged in multiple threads of 
different conversations with different 'types' of buyers. The simplicity of setting up a blog on the hosted systems generally preferred by the hosts makes creating additional blog pages easy and more importantly a hosted system also helps to manage the styles and themes that are applied across an entire Blogshop. Constructing a separate 'terms and conditions' blog is an example of how the hosts have independently developed an understanding of the need to reduce repetition and maintain consistency across many individual blog pages. By linking to a separate terms and conditions page their consolidated capture of experience and knowledge is fully applied to all transactions within the Blogshop rather than requiring duplication within each separate department. Thejewelhaus takes a slightly different approach by offering a single image that is hosted on photobucket.com to cover all of its terms and conditions (Figure 1). 


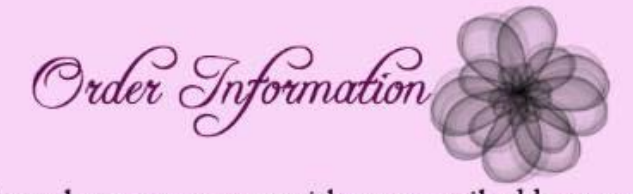

* To order an item, leave a comment with your email address or email me at thejewelhaus@gmail.com and state the item that you wish to purchase.

${ }^{*}$ Comments are screened to ensure the privacy of our customers.

* Once you receive a confirmation email, it is considered as ordering the item.

*If you change your mind after ordering an item, please notify me. Backing out at the last minute without notifying me would cause you to be listed on the Dead Buyers List.

*I do not do meetups so payment is made via ibanking and bank transfer

* For ATM fund transfer users, please provide a clear snapshot of your receipt.

* Payment is to be made within 48 hours after the confirmation of your order.

* If payment is not made by then, the order will be cancelled and the item will be put up for sale again.

* Strictly no refunds.

* Prices stated do not include postage fees.

${ }^{*}$ Normal postage costs $\mathrm{S} \$ 0.50-\mathrm{S} \$ 1.00$

* Registered postage costs $\$ \$ 2.50$

* I will take no responsibility for the loss of any items.

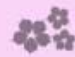

Figure 1: The terms and conditions of thejewelhaus.livejournal.com that is an image hosted on photobucket.com.

Blogshops often incorporate a 'wants list' of desired items as a side panel to their main blog or as a separate blog entirely. The items wanted are usually the most recent fashion items and hard to obtain or exclusive brand name lifestyle accessories but often more mundane needs are also listed such as 'a black bag'. Wants lists on Blogshops are not confined to those items wanted by the host herself and can extend to include sisters, 
friends and sometimes other buyers. As an example of a general 'want' on Wednesday, July 16, 2008,

Wants
URGENT!

Looking for Hei Tang Qun Xia Zhuan (Legend of Brown Sugar Chivalries) collectibles; OST, pictorial, the like. Below SGD 30, or swaps would be great!

Please email fahrenheitstuffs@hotmail.com if you have these items, thank you (: (from fahrenheitmerchandise.blogspot.com/2008/07/urgent-looking-for-dbsk-five-in-

black.html).

The collective wants lists point to the (sometimes unintentional) cooperative nature of many Blogshops and the ways in which social networking activities are intertwined and complemented by the physical acquisition of desired items. Hosts and buyers are drawn together to exchange and buy goods outside mainstream circuit of Singaporean shopping but are simultaneously drawn into a broader set of practices that results in mutual trust through star buyers and sellers lists and mutual distrust by sharing 'dead' and 'MIA' buyers lists. All of these lists refer to the buyers and hosts by their online identity and presume that while the identity is assumed by the individuals themselves it will remain immutable across time, separate blogs and multiple transactions.

A further feature of many Blogshops is the use of handmade items including cards, jewellery and ceramics (for example jjsshoppe.blogspot.com) - with the occasional hint that the facilities of the hosts' college were involved - as well as more unusual items such as cakes (bubblegum-x.blogspot.com).

Dear Prince \& Princess...

http://photos1.blogger.com/blogger2/3869/4312/1600/264a.0.jpg[264] Handmade "Purple Outer Space" Swarovski Earring A brand new pair of Handmade Earring. All my earrings will be mailed and carefully packed in bubble bag to prevent any damage. This pair of earring is made with Authetic Swarovski Crystals 4mm (Violet Opal, Light Amethyst \& Amethyst) and Cat's eye oval beads. Condition:Excellent.

P\&H: FREE LOCAL NORMAL POSTAGE

Selling @ S\$4 /- (Sold Out) 
(from www.babygodz.blogspot.com accompanied by a photograph of the earrings on a wineglass).

Handmade items reflect the relationship that hosts have to mainstream economic practice. By applying their own labour to relatively low cost and readily obtained materials additional value can be realised and this can then be applied to buying higher value and more highly desired commercial brand name items. Many aspects of the most established Blogshops include references to one or more of the colleges or high schools in Singapore and indicate that hosts come from generally financially secure family backgrounds. Their activities reflect the frustration of most teenagers who do not have direct access to disposal cash but rather to the accoutrements of middle class backgrounds such as, for instance, a college with a pottery kiln as well as digital cameras and mobile phones. All of these features reinforce the additional networks that bind and shape the design sentiment adopted by Blogshop hosts and their imprecise roles that never constitutes them as designer but similarly they cannot simply be described as a 'user'.

We're the lady bosses LYDIA and VALERIE . We're going on 14 and we study at RIVER VALLEY HIGH SCHOOL. You can make enquires at the tagboard, mail us at twocarrots-@hotmail.com, or sms or valerie [912510\#\#] / call lydia [822284\#\#] For meetups already scheduled at the east side, contact valerie. West side, contact lydia. meetup available @

\{foc\}

(at our convenience)

mrt stations:

-woodlands

-yewtee

-choachukang

-boonlay to bugis

-habourfront

others:

-outside RVHS

-queensway shopping center

you can mail us to request for other locations :D we'll consider . we are nice people :D you might have to pay a small sum though .

Please do not be late, latecomers will be charged \$1 for every 10 minutes late .

Also, if you wish to change the meetup time or place please inform us at least 4 hour earlier .

meetup twice, once for payment, once for collection.

if you only want to meetup once; want us to pay for you first, state in the order form. We'll consider, depending on whether we're rich or poor at that point in time; $D$ You'll 
probably have to pay a small sum - also depends on how rich we are then ;D (twocarrots.blogspot.com)

Each Blogshop is hosted by one or two female teenagers generally still at college. This affiliation with full-time education is repeatedly confirmed during exams periods while hosts take a break to undertake ' $O$ ' level or higher level examination preparation. For example, "We are also getting few instocks ;] Bag spree \#2 is opened now, closing on 8 oct, sry, but tht is when my exam is over. getting more designs too =D" (www.reminisce$x 3$.blogspot.com). The connection of the hosts with individual education institutions also reveals that they have an immediate network of buyers who in many cases are also hosts - which is supported by very close proximity and allows the school grounds to act as a trading floor. Posts in Blogshops refer to these practices in ways that assume relatively good levels of inside knowledge regarding the daily routine of the college as well as specific locations on campus. What is equally evident from the messages and information on each Blogshop is the relationship of this 'virtual' operation to one or more of the stations physically located on Singapore's Mass Rapid Transport (MRT) system and the integrated use of mobile phones to confirm arrangements and finalise sales and purchases. The use of the public transport network enables hosts (as a buyer) to reach buyers (as hosts) outside the closed circuit of her own college and interact with buyers and hosts associated with other colleges who may hold slight variations of fashion preferences and priorities. The procedures for obtaining items purchased from a host through the MRT network, the need to conform to her delivery arrangements and the conditions associated with pickups including payment penalties for a late appearance, and wasting a host's time, are very detailed and specific. The stringency and precision of the conditions, particularly on those that are longer established, reveal one of the ways in which Blogshop design has continuously evolved through personal and newly learned experiences and the means that the host employs to ensure that she will enjoy some form of benefit from any exchange - even if this is simply to minimise the time and hassle spent disposing of items she no longer wants and to which she has ascribed only minimal economic value. The changing 
personal circumstances of the hosts can invoke a change of terms and conditions. For example, one host only does exchanges in one MRT station during weekdays, which is close to her parent's home; however, on weekends her MRT station of choice changes to reflect the fact that she spends weekends with her grandmother in a different part of Singapore. Individual hosts have also developed the design of Blogshops from solely one of one to one exchange. The most common trajectory has been the development of a separate spree blog. The spree is a mechanism by which groups of buyers can introduce new and hence more highly desirable items into the relatively closed circuits of Blogshop exchange. By subscribing to a spree the buyer gives money to a host who then places an order with an offshore wholesaler and then once they are received redistributes the items to the subscribers. While the most obvious means for personally benefitting from this exchange is to place a mark-up on the amount each subscriber puts into the spree many organisers do not take a monetary profit but rather use the additional funds to buy items for themselves effectively gaining the highly desired items for little cost other than the time it takes to organise a group of friends, associates and trusted buyers.

Sprees are successful and popular with hosts and many Blogshops specialise in this form of exchange - with, again, specific and clear terms and conditions - as they have the benefit of utilising bulk meetups at MRT stations to distribute the goods and have already received an upfront payment from subscribers. But even this specialism can force a further iteration in design of the Blogshop as the host recognises the need, once again, to dispose of items as they become unfashionable.

The various pulls of different social and cultural networks both online and within the daily experiences of Singaporean high school life ensures that the aesthetic aspects of the Blogshops are not particularly distinctive, they retain the general look and feel of an 'amateurish' teenage blog long pages bloated with images inappropriately over-sized for webdelivery. However, this approach is now employed by more mainstream and popular blogs where less consideration is given to load times and 
there is an increasingly active resistance to the multi-page advertising laden above-the-fold approach adopted by daily newspaper and print magazine Web sites (Nielsen, 1999). Many of the images for the Blogshops are taken from mobile phone cameras and are not resized, optimised or even re-orientated. Hosts regularly also make regular use of third-party free photo hosting sites such as photobucket.com to host their images. Blogshops are further complemented with tools such as cbox.ws, a chat and tagging service, as well as code from javascript.nu and dynamicdrive.com, which are both remotely hosted Javascript libraries. The Blogshop technically becomes a front end to a collection of tools, capabilities and systems that are brought into service by the host as required. As the sophistication and size of the Blogshop expands the need for third-party subsystems to control navigation and maintain consistent styles becomes increasingly necessary. Other elements are drawn upon to craft and provide the expected elements of all Blogshops including link lists, shout boxes as well as kitsch effects such as black snowflakes falling down the screen (taken from www.24fun.com). Longer established Blogshops tend to adopt cleaner design principles and more disciplined use of imagery as well as more diverse hosting of photographs.

A feature of Blogshops distinctive from more conventional blogs or eCommerce sites is the levels and forms of personal identity being revealed. Hosts include photos with obscured faces, utilising hands and legs as the platform to model available items (Riegelsberger 2003) (Figure 2). In contrast, buyers will include 'snapshot' images of themselves and their friends at a range of identifiable locations including restaurants and daytrips. Buyers and hosts of newer Blogshops also offer commentary and observations about their daily lives. Hosts also make 'liberal' use of images taken from printed and online fashion catalogues and other Blogshops to complete their design. The scanned and copied images are undoubtedly a 'budget' and home brew approach to showing their available items. These design elements mimic strategies employed by conventional eCommerce to facilitate trust by creating an association with the prestige and quality of the brands displayed, however, the choice 
of imagery reflect the personal preferences and currently owned items of a host rather than forming a design strategy. One of the few examples of hosts incorporated in full length unobscured photos was myrunwayboutique.blogspot.com where all three hosts include full length pictures and also reveal that they are students at the National University of Singapore making them among the oldest hosts that can be identified. This Blogshop includes (in May 2009) photographs of Lady Gaga with one of the hosts imitating the pose in a matching dress creating an association with popular media celebrity and the clothes available. The Blogshop also constructs an arbitrary classification scheme for a series of popular houndstooth dresses with the red version described as 'love', the black version as 'hate' and brown as 'apathy'. With the exception of the first term these are descriptions rarely used for selling fashion items. Within the mainstream fashion and fame conscious teenage culture that the majority of Shopblogs exist there is a clear temptation to 'be' or become a model. Association of individual desired fashion items with the commercial imagery being displayed encourages this ambition and further reinforces the desire to mimic. At least, in part, as a consequence of the desire to imitate and 'be' models many hosts include 'mirror photos' - photographs they have taken of themselves with the aid of a mirror. The results are variable but generally poor; "sorry it's unclear, my camera was dying" (clearplease.blogspot.com). The use of mirror photos by the hosts in Singapore is itself an naive technological adoption and repurposing of youth culture activities found elsewhere such as the US and UK where 'sexting' (Muscari 2009) is a more common use for mirror photos in which teenagers take sexually explicit photographs of themselves and text them to friends. 


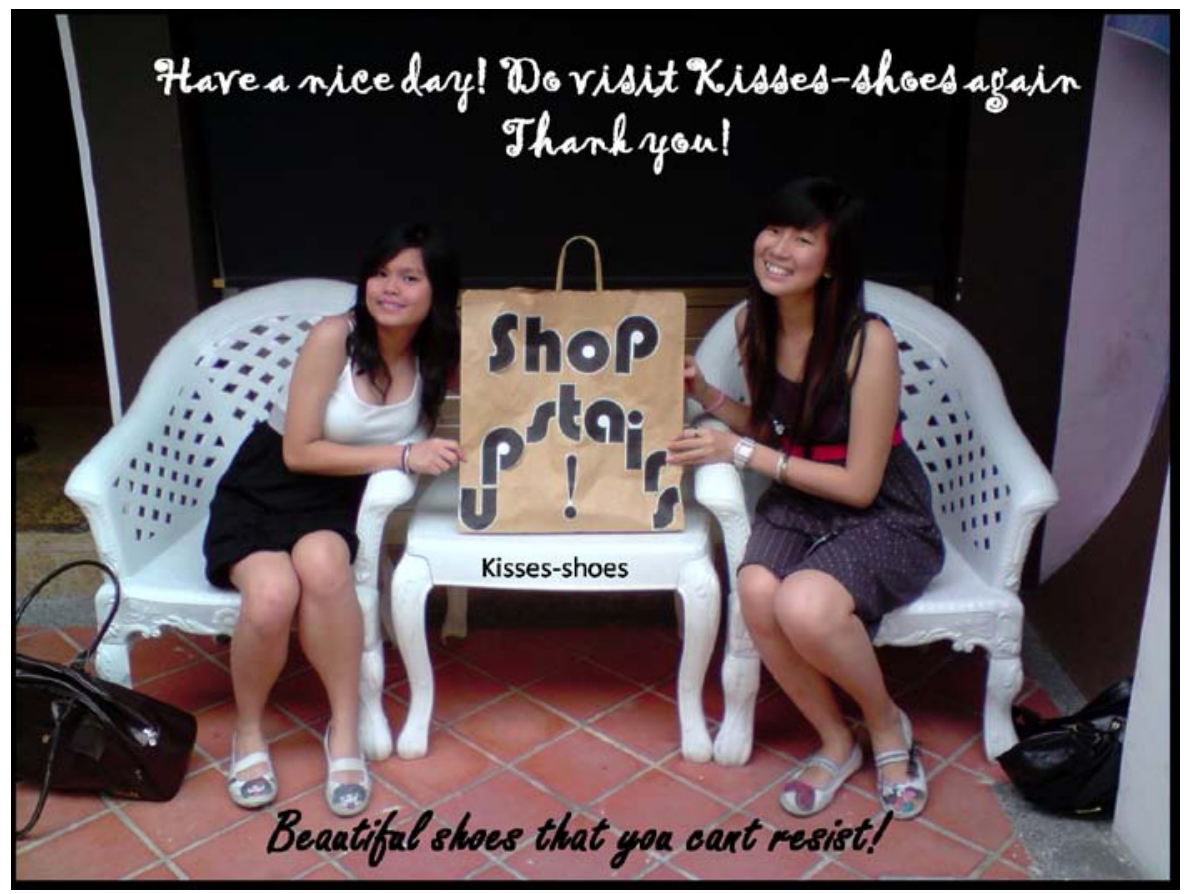

Figure 2: kisses-shoes.blogspot.com adopts the advantage of introducing handmade items in the Blogshop exchange circuits and describes the host as "I dont make shoes for a living, i am a full time student, part time shoes Maker. $=$ )" and reveals less reluctance to show her face than many hosts.

Singaporean Blogshops are hallmarked by their close relationship to the Singaporean Mass Rapid Transit (MRT) public transport system that covers the island and is the mechanism for many physical exchanges.

Meeting up is a contentious activity for hosts with many expressing complete distrust for them or at least presenting such an onerous set of terms and conditions that would discourage all but the most enthusiastic buyer. Some conditions are relatively polite such as "No meetups/reservations as we're really busy these days" (community.livejournal.com/sgflea/34572582.html) or "NO meetups, unless there is really a need." (littlehappy-shop.blogspot.com). Hosts' enthusiasm for spreeing is also partially explained by this distaste for individual meetups as the mass meetups conducted at the end of a spree enable many exchanges to happen at the same time in a context where 
the buyers (who have already paid) must meet the conditions of the host in order to receive their ordered items. None of the hosts acknowledge the risk to their personal safety in conducting an individual meetup with an unknown buyer and this reveals the tacit support provided by the broader networks complementing the social networks of Blogshops as well as the authoritarian form of Singaporean government that embeds greater provision for protection of personal security and policing presence. Where a host is prepared to meetup with buyers they are generally prepared to travel to only one to three MRT stations for the free delivery of items (FOC). Additional information offered on the blogs such as the high school they attend or the stations they will travel to for free changes on weekdays shows how closely the location of the 'free delivery' stations relate to hosts' own homes. A smaller number of hosts are prepared to travel further for free. However, this generosity should not be interpreted as client orientation or an attempt to gain competitive advantage over rival hosts but is more mundanely connected to the possession of a different travel pass and is consequently an insight into slightly different lifestyles of individual hosts. The MRT system also enables hosts to break away from the network of a single college or high school to take advantage of neighbouring networks of Blogshops to acquire rarer items not easily found within these closer networks. The rapid development of Blogshops across Singapore and with so many distinct exchange networks prohibits discovery of the original source although some Blogshops indicate that they originally had a connection with the National University of Singapore's (NUS) Students Business Clubs. And a recent posting on a discussion board about \$SG 2,000 fines being applied to making trades over the MRT fare barrier bemoans, "And yet our govt encourage us to be entrepreneurs." (www.sgclub.com/singapore/pay_goods_over_136371_3.html) highlighting the important cultural influences being received from education and government by potential and current hosts to adopt a spirit of free entrepreneurship. The earliest Blogshop that can be definitely dated is daisyloves.livejournal.com - "Want to be like her?? Korean Big Piece Clip On" and on her Bio, "Hi! I am a heavy shopper who 
buys clothes on impulse. They are either all brand new or worn less than 2 times. My aim of opening this blogshop is to lessen my closet load as it is really too packed. No intentions of earning profits at all. So $i$ am selling them at very cheap prices. $U$ take a fancy in it, u buy it. :)" - from June 2005, however, the majority of Blogshops have starting dates from 2007 onwards. Blogshops at this point in their early development clearly identified a social need and desire rather than the discovery of an unrealised technological capability hidden embedded within blogging systems. The longer term advantage of using both livejournal and blogspot has been the longevity of the systems themselves, the level of support available and the active development that continues on the themes/skins available to novice users. Both systems are owned by large companies, in the case of the latter it is Google, and have been in continuous development since their public release in 1999 (www.livejournalinc.com/aboutus.php\#ourcompany; www.blogger.com/about). All of these features would be identified as positive points of comparison for the selection of a system through the Open Source Maturity Model (OSMM) or similar approaches to selecting software candidates in the open source domain.

\section{x.3 New eCommerce?}

With over four hundred Blogshops in existence by the end of 2008 the phenomena is developing rapidly and continuously adapting. Over a relatively brief development trajectory of 2 to 3 years Blogshops reveals a common evolution of individual experiences, including technological and design experience, from a 'simple' blog as a personal but public diary into a Blogshop to the point of either complete abandonment as a shop, development as a more conventional online shop (and in at least one case a physical shop) or to the role of wholesaler supplying other Blogshops. Blogging system preference is largely the result of self-selection as both the most popular options were already popular in Singapore with the 
ability to easily link images from third party services providing an additional benefit (Nardi et al. 2004). Both blogging systems offer large amounts of design flexibility enabling relatively novice users to customise and personalise their blogs with readily available tools to include mobile phone camera images and stock clipart. Further advantages include the relative small amounts of 'competing' commercial advertising added by the systems themselves and pre-built templates that offer minimal barriers to full participation as a Blogshop host. The earliest, most experienced hosts further enforce this preference for particular systems by refusing to link out to other Blogshops hosted anywhere else effectively developing networks of Blogshops that offer, very broadly, a degree of overall navigation and structural consistency. The rationale for maintaining only intra-system links is also an unexpected type of trust formation that presumes Blogshops hosted anywhere else may be 'commercial' operations.

Just as the distinction of designer and user is problematic within Blogshops the distinction between host and buyer is not a clearly demarcated difference and individuals adopt multiple identities simulataneously. Buyers become hosts through a slow transition of practice that is not mutually exclusively.

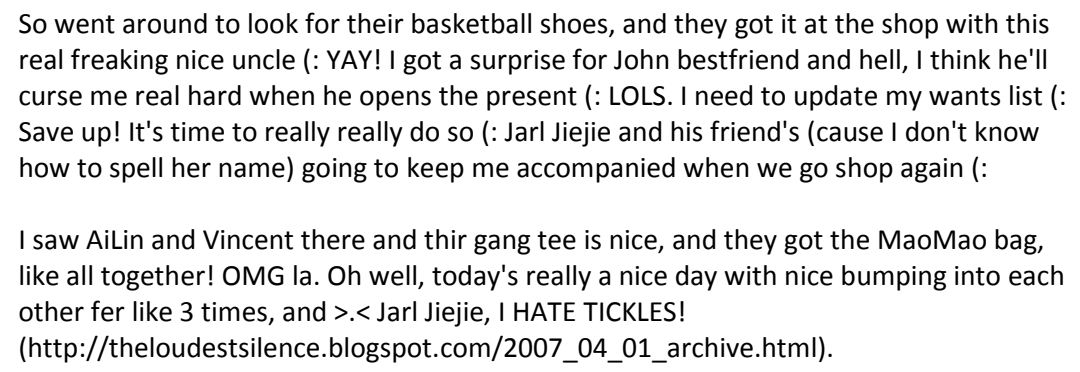

So went around to look for their basketball shoes, and they got it at the shop with this real freaking nice uncle (: YAY! I got a surprise for John bestfriend and hell, I think he'll curse me real hard when he opens the present (: LOLS. I need to update my wants list (: Save up! It's time to really really do so (: Jarl Jiejie and his friend's (cause I don't know how to spell her name) going to keep me accompanied when we go shop again (:

I saw AiLin and Vincent there and thir gang tee is nice, and they got the MaoMao bag, like all together! OMG la. Oh well, today's really a nice day with nice bumping into each other fer like 3 times, and >.< Jarl Jiejie, I HATE TICKLES!

(http://theloudestsilence.blogspot.com/2007_04_01_archive.html).

Over time buyers add offers to sell items such as jeans or $\mathrm{t}$-shirts as posts between more regular diary-like messages. Thlittlepig.blogspot.com offers a series of photos of goods that are a collection of things 'seen' rather than items offered for sale, however, the form and style of the page is one of a Blogshop and all that is missing is 
the invitation to buy one of the items captured in the photographs. In some cases the items for sale are referred to as having appeared in a previous photo being worn by the host. While a use patina is sometimes desirable and fashionable in clothing such as jeans and some types of tshirts as well as jewellery these self-referential observations between postings, perhaps initially unintentional, offers proof to the potential buyer that the host had at least at some point actually possessed the item they are attempting to sell and by implication have also assumed the role of buyer. But equally importantly for the primary audience of the host herself these posting document her evolving fashion sense and tastes in a photographic journal - the original intention of the blog itself.

Topshop inspired boho dress

Worn once, selling for \$19 (Bought for \$27)

SWAPS AT 27 (threeofbroccoli.blogspot.com)

This is not 'trust' in the consciously 'designed in' sense of existing eCommerce literature (Harrison McKnight et al. 2002) that is crafted through hallmarks of authority but is nonetheless another of the unintentional - methods of establishing trust around an individual Blogshop and its host.

An initial indicator of the self-oriented design focus of Blogshops is the tone and style adopted by the majority of hosts. While 'true' Singaporean English can be excruciatingly obtuse or unintentionally witty to a non-native reader the majority of hosts employ a form of language that just appears quirky to those outside Singapore and reconfirms they are from more privileged backgrounds (Crystal 2004, 508 \& 522). Individual usage of Singaporean English, as with elsewhere in the Englishspeaking world, is linked to socioeconomic status and ethnicity (Crystal 2004, 400). Newer Blogshops show increasingly complex and confusing phrasing that suggests their development has now been more widely adopted across a broader social spectrum of Singaporean teenagers. The expanding numbers of Blogshops is beneficial to all hosts in Singapore as the range of networks across which goods can be exchanged is increased and this presents greater opportunity for the disposal of unwanted 
second hand items at higher prices into alternate circuits where the item may still be fashionable or even emerging as fashionable. This benefit however can only be realised by the host if they can find buyers who are in more than one network or at least become buyers outside their most immediate network (Castells 1999). As this necessitates negotiations with people outside familiar networks such as friends, college or geographic proximity the language of personal accumulation is combined with phrasing more often found on formal eCommerce sites (Figure 1). The 'terms and conditions' still remains focused on more local exchange circuits (of friends) and much of the quirkiness of phrasing and terms comes from the use of local references and slang.

\footnotetext{
For those who are studying or working in Rivervale Primary School, you can collect your items in school. However, you would need to pay first. But you can pay in school :) (joycefashionshop.blogspot.com).
}

Preorder on Wallets \& Mp3 Players! Mix and match with up to 50 or more designs to choose from. And all preorders just cap at a small amount of 5-10! So obviously it isn't a long wait! :D Alright, so let me see, the Mp3 players are really a good buy. Its imported from Korea! iRiver leh! I think everyone knows that brand right ${ }^{\wedge} \wedge$. Andand, its unique hor! :D Go take a look okay! :D Muahahaha(: (hotand-cold.blogspot.com).

However, these authoritative statements are often combined with a sense of playfulness and ironic frustration. Comments such as "Im so so annoyed, some people just got ALL the time in the world to make others feel their fake sincerity before they totally disappear!" and "I emphathize very STRONGLY on TRUST\&HONESTY. I'm really nice if you respect me, \& rest assure I don't bite (: I appreciate fastdeals lots! (: To have a better idea of the sizes I usually wear; I'm a UK6-8 (mostly UK6), height $160 \mathrm{~cm}$, size 6 for feet by the way. I'm a VERY impulsive buyer and am a sucker for livejournal clothes, hence the need to clear out my wardrobe as some clothes don't fit/suit me nicely."

Such intimate reflections all disgress markedly from the phrasing of conventional top down eCommerce. The conventional presumption that a website designed for sales will speak (politely) to a global audience 
is largely absent from hosts' statements. However, immediate contradictions are revealed when hosts who are caught up with the need to break away from their safe networks that have been constructed through the security of immediate social networks deal with strangers who, if hosts' posts are to be entirely believed should never be trusted. Hosts' 'terms and conditions' blogs present their accumulated knowledge about (bad, unknown and more distant) buyers and their peculiarities with some terms referring to individual incidents that the host has no desire to experience again. This further reinforces the presence of the host herself as the primary audience and the use of the blog as a way of reminding herself of these bad experiences. This narrative of poor host experience is sometimes complemented with an additional black list of buyers and hosts with no opportunity for the accused to defend their actions.

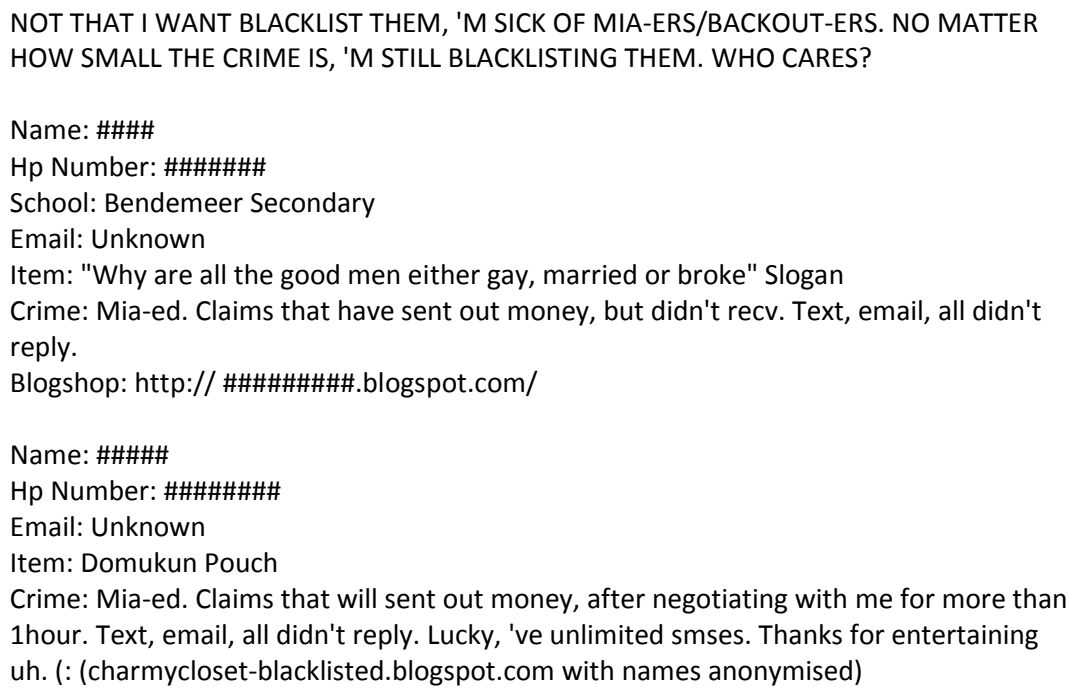

Disrupting the formal top down eCommerce design Shopblogs' "term and conditions" have become a very public knowledge management tool that informs other hosts and buyers. These common features of Blogshops constitute them as a harbinger of grassroots management and exchange that problematises the possibility for participatory commerce that is regularly claimed as democratic (Milward 1996). What is offered by 
Blogshops is a wide social and economic network of teenagers in Singapore that are composed of a collection of smaller more personal networks that are at the core of each host's network of friends and relatives. Iterative and polymorphic design practices enable these teenagers to take full advantage of a wider range of smaller networks to maximise their individual advantage. Irrespective of the closeness of personal connections that are drawn upon by the hosts they are driven by personal motivation (boyd and Ellison 2007) and take advantage of the small variables of fashion preference that can exist even in a small geographic region such as Singapore. By attempting to offer a parallel to conventional down-the-line trading of contemporary capitalist supply chains (Thrift 2005) while wanting the items of fashion crafted by this system the need to profit from trading is subsumed by the hosts to the more fundamental desire to possess.

\section{x.4 Blogshop Hegemony}

Blogshops enable a form of technologically mediated exchange which presents a resistance to prevalent forms of capitalist hegemony regularly experienced online and directly within Singapore. For both the hosts and buyers the Blogshop encourages commercial resistance, forms of recycling and shifts emphasis of consumption practice away from 'sites' of mainstream shopping. Blogshops also in this way alter the usual seller 'producer', buyer 'user' dichotomy so often presumed in online shopping exchange (Friedman et al. 2007). For the host their motivation is focused around acquiring fashion items that are currently popular with the host and her circle of friends and disposing of those items that have fallen out of favour within the same circle. The host in this way transcends the disassociated role of producer who conducts their activities with profit as primary motivation (Cormode and Krishnamurthy 2008). Friends are only one of the influencers that determine what items are fashionable with wider circles of fashion referenced throughout the postings. For example, 
"We have the latest trends and fashion imported directly from Japan, Korea and HongKong" and "30-40\% OFF RETAIL PRICE!! BRAND NEW, Specially Imported(not available at local stores) from mainly JAPAN $n$ KOREA with some DESIGNER APPAREL, only 1 pc available unless stated!" are postings from missydixie.blogspot.com and shopping-fortrend.blogspot.com respectively. On charmycloset.blogspot.com the situation is made clear. The following blog post not only reveals the cultural pressures that the shifting foibles of fashion bear upon the hosts but also the ways in which conventional notions of profit are heavily mediated and subsumed within the Blogshop environment.

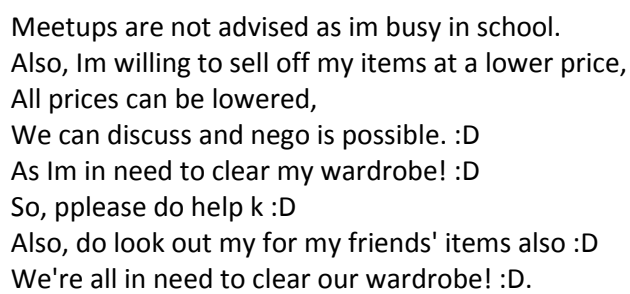

Many of the Blogshops stress that their second-hand clothes have been worn 'only once' or 'lightly' by them but few references are made as to whether these items were bought new or from another Blogshop. The host at ruthneedsmoney.blogspot.com offered a dress that was subsequently sold with the price difference representing an intermediate rental cost to the host.

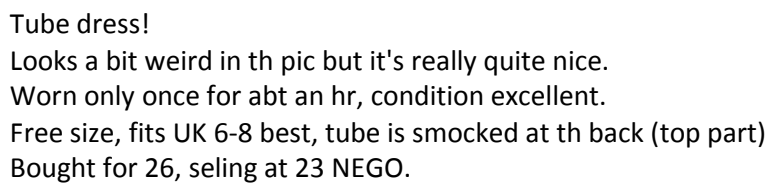

The unconventional business practices of the hosts reveals a form of capitalism that is orientated around the temporary accumulation of goods and the iterative possession of specific symbols of prestige (visual, technological and spatial to name but a few). In many situations the exchange also invokes a form of ongoing obligation between the host and 
her buyers. Disentangling the distinctions between users and designers (Lamb and Kling 2003) does not suggest that Blogshop hosts are simply engaged in forms of gift and commercial exchange but rather that they are taking advantage of 'social networking' and even the implied cultural obligations built into these networks in order to obtain personal benefits that are measured (currently) by having skinny jeans and Cookie Monster t-shirts rather than pounds or dollars. The Blogshops are designed to enable a particular teen lifestyle for the host herself. The mash-up of personal life revelations, mirror photos, borrowed commercial images and transportation requirements reveals the technology enabled environments of a generation capable of designing the means to suit their desired lifestyle.

For the buyers the benefits of Blogshops may appear marginal such as slightly cheaper clothes, jewellery or makeup than mainstream shops or the convenience of a more local pickup for hard-to-get items. However, it is important to not overly disentangle 'being' a host from activity as a buyer as the two roles are necessarily inter-related. Buyers who are 'good' or easy to deal with will often be named in the blogroll - a common design feature of blogs turned to a different purpose - and this can often act as a passport to enable purchases from other hosts and other exchange networks. The practice of exchanging items at a college suggests that 'buying' has also become a form of schoolyard 'play' that is preparation for excursions to further flung networks and different items of desire. The various descriptions of exchanges at MRT stations can vary from a social outing to something akin to a spy mission in enemy territory making the act of buying itself as much the focus of social activity as the actual possession of any specific items. The most intangible rationale for buying is that it provides content for blog postings. This crafts the blog with dual purpose. It provides the mechanism for arranging the exchange of physical items and offers reasons to form and maintain a social network. Equally importantly the act of exchange provides textual and visual content for the blogs as well as still further items to exchange. 
The general reluctance to conduct straight 'swaps' of items could be interpreted as an attempt to generate profit from exchanges - as a financial transaction is required to obtain items. But it is the influence of fashion, issues of trust and the hosts' own motivations that mitigate this as the sole rationale for the 'no swaps' policy of many Blogshops. In the relatively closed exchange networks of the Blogshops the range of items available to swap will only produce marginal benefit for the host as what is available will only fall within a narrow range of acceptable items.

Exchanges outside the trusted network of a college or similar location increases the possibility that there is no recourse to compensation for a 'bad' swap and consequently emphasising the need for a social network to name and blacklist 'bad' buyers. With a host insisting on money - as a known item of recognised and consistent value rather than just a mechanism for accumulating profit - for the exchange they remain "in charge" of the exchange and minimise their own personal risk.

\section{x.5 Designing trust - and the love}

Blogshops increase a host's economic independence and improves their cultural standing among peers by provisioning them with the latest fashion items and desirable rare goods. While this may appear trivial motivation it is indicative of a fame obsessed culture that is, encouraged and shaped by the ready and rapid dissemination of large of volumes of gossip and imagery through the Web. This is further reinforced by the intersection of cultures represented in Singapore which in effect becomes a point of convergence for a global range of attitudes and understanding (Jones 2007, 450). Blogshops formed from conventional blogging activity (Nardi et al. 2004) with the site listing a set of desired clothing or items that were superfluous to the host's requirements. Most hosts were buyers first and continue to buy. In this environment, Blogshops are a form of eCommerce crafted without the nuances of conventional online shops that addresses the desires of teenage girls to possess fashion items. 
The bias of the 'terms and conditions' on Blogshops are entirely in the hosts' favour and authored to benefit their desires. Despite being a key feature of Blogshops the terms and conditions also reveal that meetups are a problematic distribution method for hosts with each offering different solutions. The majority of hosts complain about buyers wasting their time by being late or not showing up to exchange goods and money. Many insist on the buyer having a valid or pre-confirmed mobile phone but far more incorporate the right to charge late fees in generally blunt tones. Robot and Girl (duabui.blogspot.com) says "If you are late without telling me beforehand, a penalty of $50 \mathrm{c} / 5 \mathrm{~min}$ will be charged." Cutiesweetieslovelycloset .blogspot.com demands a "\$10 charge for cancellations within an hour. \$1 will be charged per minute of lateless after 10 minutes of waiting." Xkimiirawrrs.blogspot.com takes a different approach by offering to deliver directly to the buyer's door if the buyer is prepared to pay the two-way cab fare. The onus is very much placed onto the buyer to be in the right place at the right time implicitly reflecting an underlying cultural belief in the efficiency and security of the MRT and public transport system of Singapore. Ultimately for many of the hosts as their Blogshop has developed and expanded many now refuse to do meetups of any sort and will only work on the basis of posting an item once payment has been received - in effect, a conventional eCommerce model for overcoming issues of trust or rather its lack (Essler 2001). The responsibility for establishing trust rests entirely with the buyer. Despite the proliferation of Blogshops few hosts have also adopted more conventional approaches for establishing trust by, for example, advertising the length of time they have been operating, instead hosts have chosen to use claims such as that used by awesomehighway.blogspot.com, "i am nice i don't bite." The necessities of crafting trust do not obliterate the central premise of Blogshops to benefit their hosts and yet a number of the better established sites are now claiming to be making a lose. The conventional balances and relationships of communicative authority and commercial power to personal consumption have become altered with Blogshops. The terminology of Blogshops reveals this sentiment with a blend of bloggers terms such as 
MIA-ing (missing in action) and wholesale terms such as FOC (free of charge). Hosts are imitating the tone and language of conventional commerce but remain motivated by a desire for consumption and possession without the mediating process of accumulating monetary profit in order to buy goods commercially. At an operational level this means that each additional cost associated with an exchange is directly and transparently passed onto the buyers including transport, the cost of labour (expressed through the time a host waits at an MRT station or the cost of turning raw materials into a desirable item) and loses incurred by non-payment of goods. Few Blogshops offer a single total cost for purchasing an item (as would be done in conventional commerce or eCommerce) making the exchange error-prone and offering (too) many opportunities for buyers to negotiate.

At Oreocheesecake.blogspot.com "SWAPS are acceptable, unless stated otherwise" but this generosity is moderated by the additional statement that "If you initiate the swap, it will have an additional charge of $\$ 3$ on top of the stated price for the particular item." effectively making it unclear as to whether the exchange is a swap or a purchase. Similarly at crownedauctions. blogspot.com, "SWAPS ARE WELCOMED(PROVIDED THAT I AM INTERESTED IN YOUR ITEM)." and "PRICES OF ITEMS ARE INCREASED(STATED) WHEN DOING SWAPS REGARDLESS OF WHO INITIATED IT."

The language of Blogshops has shifted over time towards imitation of retail outlets such as those at cometomyshop.blogspot.com where "ALL PREORDERS !!! all clothes are 100\% material guarenteed !!" and mis-matchnmix.blogspot.com where "for any enquires or orders all items are BRAND NEW otherwise stated price are NON-NEGO as they are of the lowest we can offer NO SWAP is allowed all price are EXCLUDE POSTAGE." Many Blogshops focus emphasise on goods that are currently trendy in respected but exotic destination such as Hong Kong or Japan. This trajectory differs from earlier attempts to solely divest the host of their second hand items. 
These developments suggest that there is a progression through the roles of 'buyer', 'swapper' and 'spree-er' to host and onto the roles 'shopowner', 'wholesaler', 'MIA-er' or 'clearance seller'. However, this interpretation can only be applied when a single site is viewed in isolation, as an individual will be active across a network or series of networks.

This chapter shows Blogshops as a polymorphic and iterative form of eCommerce practice that is motivated by a desire for consumer goods initiated without the mediating process of accumulating profit. Organic user-oriented design found in the grassroots ecosystem management (GREM) of online shops, challenges Canas' (2009) assertion that designers work in academic or industrial environments. Examples provided in this chapter illustrate how the intersection of Web2.0 tools with self motivation result in complex technologically-enabled social exchanges. In the cases explored here Blogshops enable a capacity for direct conversation and aesthetic expression through social media to produce unexpected empowerment. The rapid development of Blogshogs has enabled a commonality and evolution of individual experience enabled by a rich assemblage of technology, culture and location that offers a first level insight into a complex environment that brings together this form of economic need, locational circumstance, and technological capacity. Grassroots participation draws upon bottom up expertise and community knowledge is clearly relied upon and presented through these technologically enabled environments. The complexity of organising sprees, meetups and the general quality of goods offered by hosts all raises issues concerning trust and interaction via technologically enable social networks. Hosts will often reveal their personal phone number, name, general location, sometimes photographs of themselves and in a few cases their banking details (for the purpose of buyers transferring funds to them). The engagement between hosts and buyers and the range of images the Blogshops employ all reflect a complex cultural relationship that extends beyond 'simple' direct commercial exchange (Riegelsberger 2003) and often written out of more convention 
commercial design practices. Blogshops offer significant insight into a broader series of contemporary cultural experiences including the meaning and extent of eCommerce and social networking, forms of globalised youth culture, the imprecision of fashion and shifting mainstream attitudes towards self-representation. While our discussion focuses around cultural practices in Singapore, ongoing financial uncertainty in the US and in Europe offer the potential for the Blogshop to gain in popularity in a wider range of situations. Blogshops also offer the possibility for the development of the new iterative and polymorphic design practices that question and problematise assumptions already made in relation to eCommerce including the construction of trust, the distinction of the role of user from that of designer, the notion of audience and the mechanisms through which contemporary exchange is conducted.

Acknowledgements

The authors thank Donna Duncan for her assistance in identifying the Blogshops examined in this chapter.

\section{References Cited}

Beer, D. (2008). Social network(ing) sites revisiting the story so far: A response to danah boyd \& Nicole Ellison. Journal of Computer-Mediated Communication, 13 (2), 516-529.

Beer, D., \& Burrows, R. (2007). Sociology and, of and in Web 2.0: Some initial considerations. Sociological Research Online, 12(5), http://www.socresonline.org.uk/12/5/17.html.

boyd, d., \& Ellison, N. (2007). Social networking sites: definition, history, and scholarship. Journal of Computer-Mediated Communication, 13 (1). 
Canas, J. (2009). The Future of Interaction Research: interaction is the result of top-down and bottom-up processes. In P. Saariluoma \& H. Isomaki (Eds) Future of Interaction Design II, Springer-Verlag: London.

Castells, M. (1999). The information age: economy, society and culture. Oxford: Blackwell.

Cooley, M. (1987). Architect or Bee: The Human Price of Technology. London: The Hogarth Press.

Cormode, G., \& Krishnamurthy, B. (2008). Key Differences between Web 1.0 and Web2.0, First Monday. 13(6), firstmonday.org/htbin/cgiwrap/bin/ojs/index.php/fm/article/view/2125. Crang, M., Crosbie, T., \& Graham, S. (2007). Technology, time-space, and the remediation of neighbourhood life, Environment and Planning A. 39, 2405-2422.

Crystal, D. (2004). The Stories of English. Allan Lane: London.

Essler, U., \& Whitaker, R. (2001). Re-thinking E-commerce Business Modelling in Terms of Interactivity. Electronic Markets. 11(1), 10-16.

Friedman, B., Kahn, P., \& Borning, A. (2007). Value Sensitive Design and Information Systems. In P. Zhang \& D. Galletta (Eds), Human-Computer Interaction in Management Information Systems: Foundations. M.E. Sharpe: New York.

Geok, L.B. (2000). Census of Population 2000: Geographic Distribution and Travel. Singapore Department of Statistics: Singapore.

Greenbaum, J., \& Kyng, M. (1991). Design at Work: Cooperative Design of Computer Systems. Lawrence Erlbaum: Hillsdale, NJ.

Greenhill, A., \& Fletcher, G. (2009). Blog/shop: technology and grassroots management, The 6th International Critical Management Conference, Warwick Business School, 13-15th July. 
Harrison McKnight, D., Choudhury, V., \& Kacmar, C. (2002). Developing and Validating Trust Measures for e-Commerce: An Integrative Typology, Information Systems Research, INFORMS. 13(3), 334-359.

He, J., \& King, W. (2008). The Role of User Participation in Information Systems Development: Implications from a Meta-Analysis. Journal of Management Information Systems. 25(1), 301-331.

Jarvenpaa, S., Knoll, K., \& Leidner, D. (1998) Is anybody out there?: antecedents of trust in global virtual teams, Journal of Management Information Systems. 14(4), 29-64.

Jones, C. (2007). Hong Kong, Singapore, South Korea and Taiwan: Oikonomic Welfare States, Government and Opposition, 25(4), 446-462.

Lamb, R., \& Kling R. (2003). Reconceptualising Users as Social Actors in Information Systems Research, MIS Quarterly. 27(2), 197-235.

Milward, H. B. (1996). The changing character of public sector. In J. Perry (Ed.) The Handbook of Public Administration, Jossey-Bass: San Francisco.

Muscari, M. (2009). Sexting: New Technology, Old Problem. Medscape Public Health \& Prevention. www.medscape.com/viewarticle/702078.

Nardi, B., Schiano, D., and Gumbrecht, M. (2004). Blogging as Social Activity, or, Would You Let 900 Million People Read Your Diary? CSCW'04. 6(3), 222-231.

Nielsen, J. (1999). Designing Web Usability: The Practice of Simplicity, New Riders Publishing: Thousand Oaks, CA.

Ping, L., Swe, K., Hew, T., Wong, P., \& Shanti, D. (2003). Exploring critical aspects of information technologies integration in Singapore schools, Australian Journal of Educational Technology. 19(1), 1-24.

Riegelsberger, J., Sasse, M., \& McCarthy, J. (2003). Shiny Happy People Building Trust? Photos on e-Commerce Websites and Consumer Trust, $\mathrm{CHI}$ 2003, April 5-10, Ft. Lauderdale. 
Tan, J., \& Gopinathan, S. (2000). Education Reform in Singapore: Towards Greater Creativity and Innovation? NIRA Review, Summer, 5-10.

Thrift, N. (2005). Knowing Capitalism, Sage: London.

Weber, E. (2003). Bringing Society Back In: Grassroots Ecosystem Management, Accountability and Sustainable Communities. American and Comparative Environmental Policy. MIT Press: Cambridge, MA.

Whiteley, N. (1993). Design for Society. Reaktion Books Ltd: London. 